\title{
How to Personalize Pharmacoproteomics?
}

\section{Victor P Andreev*}

Department of Psychiatry \& Behavioral Sciences and Department of Biochemistry \& Molecular Biology, Miller School of Medicine, University of Miami, Miami, FL, USA

Stepping into the elevator this morning, I noticed a familiar yet strange scene: five people staring into their smart phones. To be completely honest, it required some effort not to do the same. Why are we doing this? How many of us really need to check e-mails every two minutes? Is it the modern substitute of cigarettes, a way to feel comfortable in the company of strangers? More interestingly, can we use the same psychosocial phenomenon to achieve something useful and valuable? More specifically, can we make proteomics and pharmacoproteomics "sexy" for millions of people, popularize and personalize it? The question is worth examining, since the price that 150 million [1] smart phone owners in the US pay for data plans solely (which most of us do not really need) is $\$ 30$ a month, which adds up to $\$ 54$ billion annually, or nearly twice the total NIH budget ( 30 billion USD in 2014 [2]).

Imagine the progress medical science could achieve in case some part of this eagerly and almost uselessly spent 'smart phone money' was attracted to personalized medicine instead of twitting and texting. We, human beings are naturally interested in ourselves and our health. We want to know and predict how our actions, e.g. medications, diet, training, etc. influence our well-being. Personalized pharmacoproteomics has a potential to provide us with such feedback.

The concept of $\mathrm{P} 4$ (predictive, personalized, preventive, and participatory) medicine was coined by Leroy Hood a decade ago [3] and is now advocated and advanced by many including the P4 Medicine Institute [4]. A recent paper [5] emphasized the importance of "the behavioral and psycho-cognitive components that affect how individuals act to prevent, cope and react to illness, decide about different therapeutic options, interact with health care providers and adhere to treatment" and suggested transformation of 'P4 medicine' into 'P5 medicine' with the 'fifth P' standing for the psycho-cognitive aspects. In my mind, pharmacoproteomics is an essential part of the $\mathrm{p} 4$ or the $\mathrm{p} 5$ medicine, and could be the forerunner in the process of popularization of the $\mathrm{p} 5$ medicine and in actually making it the p6 medicine, where 'sixth P' stands for 'popular'.

There are several definitions of pharmacoproteomics as reviewed and discussed in [6]. Broadly speaking, pharmacoproteomics deals with the changes in the abundances of the proteins caused by the administration of the medications, and therefore examines at the protein level "what the drug does to the body" [7], including the side effects. Pharmacoproteomics therefore can be viewed as the molecular pharmacodynamics of the 'omics era'. However, the popularity of the term "pharmacoproteomics" is still not very high. The number of matches returned by Scopus search increased moderately from 59 in October 2011 [6] to 86 in March 2014. Many of the new papers are quite important, including the pharmacoproteomics comparison of the action of warfarin and rivaroxaban on plasma proteins in patients with nonvalvular atrial fibrillation [8], the pharmacoproteomics investigation of response to antidepressants (nortriptyline and escitalopram) in mice [9], and pharmacoproteomics and pharmacogenomics evaluation of glial cell line-derived neurotropic factors as therapeutics [10]. Some other papers do not use the term 'pharmacoproteomics' and therefore are not included in the above list of 86 matches, however they report on the very important pharmacoproteomics studies, like the recent paper [11] where quantitative proteomics was used to reveal the mechanism of action of the anti-cancer drug lenalidomide, as well as the mutation responsible for resistance to this drug. The success of these papers reflect the advancements in proteomics technology that enabled "to ramp up coverage to $50-80 \%$ of the expressed mammalian proteome" and as formulated by Steven Carr "put hand over heart and say we are actually 'doing proteomics"' [12]. However, it will probably take a while before this level of proteome coverage spread from the best proteomics groups to the whole proteomics community and will be universally accepted by the broader biomedical community.

While total proteome, pursued in the above studies, is a Holy Grail of the 'omics science', some less global approaches could yield better results in the early stages of personalized pharmacoproteomics. Of interest here, is the approach developed by Roman Zubarev's group [13], where instead of depleting 12 most abundant proteins in human plasma and then analyzing the rest of the plasma proteome (as conventionally done), they concentrated on the accurate measurements of the abundances of 100 most abundant proteins, which can be quantitated much easily and with higher reliability than the low abundant ones. Importantly, the deviations of the concentrations of these 100 most abundant proteins can likely serve as predictors of several major diseases.

The extremely popular recent study of all-cause mortality in 17,345 subjects [14], which was viewed by 65 thousand persons in less than a month, communicated the similar message. This study used NMR spectroscopy to quantitate 106 candidate biomarkers (lipids, proteins, and metabolites) in 9,842 non-fasting plasma samples collected in Estonian Biobank and demonstrated that the levels of four biomarkers: plasma albumin, alpha-1-acid glycoprotein, very-low-density lipoprotein (VLDL) particle size, and citrate accurately predicted shortterm risk of cardiovascular mortality as well as death from cancer and other nonvascular diseases. This finding was validated in 7,503 samples population based cohort in Finland. Obviously, the relation of these biomarkers level with the short-term risk of death is not necessary causal. However, it is very interesting to see if and how the level of these biomarkers is influenced by the existing cardiovascular and anti-cancer medications.

The beauty of the 'p6 medicine' (and of personalized pharmacoproteomics) is that it is able to generate 'big' data and provide enough statistical power to examine effects that are missed in the smaller scale studies. The major problems with the 'popular' medicine are the sample collection and ethical issues. The consumer genetics company 23 andme successfully solved the problem of sample collection

*Corresponding author: Victor P Andreev, Department of Psychiatry \& Behavioral Sciences and Department of Biochemistry \& Molecular Biology, Miller School of Medicine, University of Miami, Miami, FL, USA, Tel: 305243 3487; E-mail: VAndreev@med.miami.edu

Received March 22, 2014; Accepted March 24, 2014; Published March 31, 2014

Citation: Andreev VP (2014) How to Personalize Pharmacoproteomics? J Pharmacogenomics Pharmacoproteomics 5: e137. doi:10.4172/2153-0645.1000e137

Copyright: (c) 2014 Andreev VP. This is an open-access article distributed under the terms of the Creative Commons Attribution License, which permits unrestricted use, distribution, and reproduction in any medium, provided the original author and source are credited. 
Citation: Andreev VP (2014) How to Personalize Pharmacoproteomics? J Pharmacogenomics Pharmacoproteomics 5: e137. doi:10.4172/2153$0645.1000 \mathrm{e} 137$

Page 2 of 2

by providing the convenient device for collecting and preserving saliva samples, but was halted by FDA based on the ethical and consumer protection concerns [15]. Personally, I was lucky to have my saliva analyzed prior to the FDA decision, and I had a very useful and pleasant discussion of the results of this analysis with my primary care physician. Hopefully, the solution to the ethical issues will be found, perhaps through the participation of the primary care physicians, which could then serve as a template for the similar solution in personalized pharmacoproteomics. The sample collection for pharmacoproteomics is not as strait forward as in genetics, since protein biomarkers are tissue specific and are different in blood and in saliva. Solutions can be sought in several directions, including the decreased blood sample volume through targeted proteomics, and the advanced discovery of saliva biomarkers. Bioproximity, LLC and Attila Csordas reported on the pioneering project in personalized saliva proteomics. It is still rather expensive ( $\$ 1000$ per sample) and requires optimization of several steps, as frankly reported in $[16,17]$, but it sounds very promising and exciting. Remember, our cell phones were ugly, expensive and were not smart not so long ago.

\section{References}

1. http://www.pewinternet.org/2013/06/05/smartphone-ownership-2013/

2. http://news.sciencemag.org/funding/2014/01/u.s.-science-agencies-get-somerelief-2014-budget

3. Weston AD, Hood L (2004) Systems biology, proteomics and the future of health care: toward predictive, preventative, and personalized medicine. J Proteome Res 3: 179-196.

4. http://p4mi.org/p4medicine

5. Gorini A, Pravettoni G (2011) P5 medicine: a plus for a personalized approach to oncology. Nat Rev Clin Oncol 8: 444.
6. Andreev VP (2011) Pharmacoproteomics of obesity: definitions, role and a case study of dynamics of human plasma proteome. Pharmacogenomics 12 : 1363-1365.

7. Benet LZ (1984) Pharmacokinetics: basic principles and its use as a tool in drug metabolism. In: Drug Metabolism and Drug Toxicity. Raven Press, NY, USA.

8. Chan MY, Lin M, Lucas J, Moseley A, Thompson JW, et al. (2012 ) Plasma proteomics of patients with non-valvular atrial fibrillation on chronic anticoagulation with warfarin or a direct factor Xa inhibitor. Thromb Haemost 108: $1180-1191$.

9. Malki K, Campbell J, Davies M, Keers R, Uher R, et al. (2012) Pharmacoproteomic investigation into antidepressant response in two mouse inbred strains. Proteomics 12: 2355-2365.

10. Park S (2013) Evaluation of Glial Cell Line-Derived Neurotrophic Factors as Therapeutics: Pharmacogenomic and Pharmacoproteomic Studies. Current Pharmacogenomics and Personalized Medicine 11: 76-85.

11. Krönke J, Udeshi ND, Narla A, Grauman P, Hurst SN, et al. (2014) Lenalidomide causes selective degradation of IKZF1 and IKZF3 in multiple myeloma cells. Science 343: 301-305

12. https://theanalyticalscientist.com/issues/0214/proteomics-for-the-people/

13. Y Lyutvinskiy, Yang H, Rutishauser D, Zubarev RA (2013) In silico instrumental response correction improves precision of label-free proteomics and accuracy of proteomics-based predictive models. Mol Cell Proteomics 12: 2324-2331.

14. Fischer K, Kettunen J, Wurtz P, Haller T, Havulinna AS, et al. (2014) Biomarker Profiling by Nuclear Magnetic Resonance Spectroscopy for the Prediction of All-Cause Mortality: An Observational Study of 17,345 Persons. PLoS Med 11 e1001606.

15. http://www.fda.gov/iceci/enforcementactions/warningletters/2013/ucm376296. htm

16. http://personalproteomics.wordpress.com/2012/03/15/personal-proteomicsreality-check-on-bioproximitys-proteome-cluster-early-2012/

17. http://www.bioproximity.com/2012/03/19/toward-personal-proteomics.html 